\title{
A symbiotic mosaic
}

Many insects that feed on plant sap can survive on such a nutritionally poor substrate owing to their association with nutrient-providing bacterial endosymbionts. In Planococcus citri mealybugs, this association is particularly interesting, as it involves a nested symbiosis in which the P. citri betaproteobacterial endosymbiont, 'Candidatus Tremblaya princeps str. PCIT' (Tremblaya PCIT), harbours its own endosymbiont, the gammaproteobacterium 'Candidatus Moranella endobia'. Writing in Cell, John McCutcheon and colleagues now reveal that this

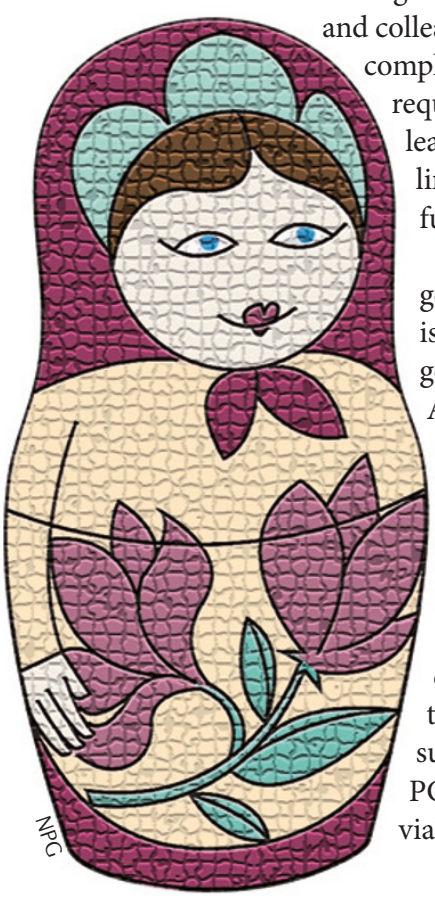
complex tripartite relationship requires genetic input from at least six different organismal lineages to be metabolically functional.

At just $139 \mathrm{~kb}$, the genome of Tremblaya PCIT is the smallest bacterial genome reported to date. Although obligate endosymbionts are known to have severely reduced genomes, the Tremblaya PCIT genome is unusual, as it lacks functional homologues for many crucial components of translation. It has been suggested that Tremblaya PCIT remains metabolically viable by utilizing essential gene products supplied by its own endosymbiont, ' $\mathrm{C}$. Moranella endobia. However, it is also possible that the missing functions are encoded by the host, $P$. citri, or that genes from Tremblaya PCIT or other bacteria have undergone horizontal gene transfer to the $P$. citri genome and the products of these genes are transported back into the Tremblaya PCIT cytoplasm.

To begin to distinguish between these different possibilities, the authors sequenced the complete genome of a Tremblaya strain that lacks an intracellular symbiont ('Candidatus Tremblaya phenacola str. PAVE' (Tremblaya PAVE)). They found that the Tremblaya PAVE genome is much less degenerate than that of Tremblaya PCIT, suggesting that the unusual degree of degeneracy in Tremblaya PCIT stems from the acquisition of ' $\mathrm{Ca}$. Moranella endobia'. Many of the translationrelated genes that were absent from Tremblaya PCIT were present in Tremblaya PAVE. Importantly, however, both Tremblaya PAVE and Tremblaya PCIT lacked the genes required for lysine and histidine biosynthesis.

So how are these essential enzymatic gaps filled? The authors used RNA sequencing to identify expressed genes in the P. citri bacteriome (the cellular structure housing the endosymbionts). This revealed 22 expressed horizontally transferred genes, including lysine biosynthesis genes, as well as genes encoding proteins involved in the biosynthesis of other nutrients and in bacterial cell wall maintenance. Surprisingly, phylogenetic analysis showed that none of the horizontally transferred genes clustered with genes from the class Betaproteobacteria. Instead, they seem to have been transferred from various members of the classes Alphaproteobacteria or Gammaproteobacteria or the phylum Bacteroidetes. The authors confirmed that the identified genes were encoded in the insect genome by generating a low-pass draft genome sequence for $P$. citri.

So, this complicated symbiosis turns out to be even more genetically complex than previously thought. The combined genomic and transcriptomic data suggest that the essential metabolic pathways in this symbiotic mosaic are completed by heterologous complementation from an extraordinary variety of sources.

Sheilagh Molloy

ORIGINAL RESEARCH PAPER Husnik, F. et al. Horizontal gene transfer from diverse bacteria to an insect genome enables a tripartite nested mealybug symbiosis. Cell 153, 1567-1578 (2013) FURTHER READING McCutcheon, J. P. \& Moran, N.A. Extreme genome reduction in symbiotic bacteria. Nature Rev. Microbiol. 10, 13-26 (2012) 